\title{
Intensity Compensation within Series of Images
}

\author{
Grégoire Malandain $^{1}$ and Eric Bardinet ${ }^{1,2}$
}

1 Epidaure, INRIA, 2004 route des lucioles BP 93, 06902 Sophia-Antipolis cedex, France, \{eric.bardinet, gregoire.malandain\}@sophia.inria.fr

2 CNRS UPR 640 - LENA, 47 Boulevard de l'Hôpital, 75651 Paris cedex 13, France Eric.Bardinet@chups.jussieu.fr

\begin{abstract}
This article addresses the problem of histogram matching in the context of medical image processing. Such a problem occurs while comparing two images of the same object, where intensity differences are due to different acquisition conditions. This can be compensated by histogram matching or equalization. To achieve this, we based our method on windowing techniques. This allows to match implicitly continuous probability density functions, yielding more robust results than the methods issued from discrete histograms.
\end{abstract}

\section{Introduction}

We address in this article the problem of intensity compensation between images taken under different conditions. This problem is part of a complete scheme where one wants to reconstruct an homogeneous image from multiple acquisitions that exhibit differences in position or in intensity. Geometrical differences can be compensated by image matching techniques [MV98], although intensity differences have to be compensated by histogram matching.

This problem can be illustrated by two examples.

- Evolutive lesions in SEP lesions can be detected in a time series of 3D images RSMA01 (4D image) by correlating the temporal signal of each voxel with an evolving lesion model. Before conducting any statistical analysis, the images have to be normalized in intensity.

- Fusion of MR and histological data (consisting of series of 2D contiguous sections) needs first the reconstruction of a $3 \mathrm{D}$ volume from the $2 \mathrm{D}$ histological slices. This reconstruction is performed by using dedicated registration techniques $\mathrm{ORS}^{+} 01$. Because of the imaging conditions during histology, the reconstructed volume is generally not homogeneous in intensity from one section to the next. Therefore, intensity normalization is needed.

These two examples are quite different. In the first one, the imaged object (here the head) is the same for all the acquisitions, thus intensity normalization can be done by comparing each image with a single reference image. In the second one, which is our main interest here, each image is only slightly different (but still comparable) from the two adjacent ones, but is not comparable with the other images because of increasing anatomical differences. Here, the intensity normalization has to be done for each pair of contiguous images, and has to be 
propagated in the whole volume. Because of this propagation, a highly robust technique is required.

Classically, histogram equalization consists in transforming the input histogram into a flat histogram Cas96. Thus, matching two histograms can be achieved by (implicitly) using this flat histogram as intermediary [Hea96]. This approach is not robust at all, as it depends highly on the extremal values of the intensity histogram, therefore it is not applicable for our purpose.

The approach developed by Wand et al. $\mathrm{WLB}^{+} 98$ is closer to our needs. They compute the best linear transformation between two histograms by minimizing a cost function (sum of squared differences). However, their approach is dedicated to MR images, the way they transform histograms is heuristic, and they need to introduce an extra parameter to minimize their cost function (due to the fact that they consider histograms and not probability density functions).

In this paper, we propose to use windowing techniques to estimate continuous probability density functions (PDFs) from discrete ones. This way, we will have a formal frame to apply an intensity transformation to a discrete pdf, in order to compare it to an other discrete pdf. This approach will be explained in the next section. In section 3 histogram matching procedure is presented. Intensity compensation in human brain optical images and monkey's brain autoradiographs are then presented in section 4

\section{Windowing Techniques for Discrete pdf Transformation}

In this section, we present all the necessary notions that will be used throughout this paper. We denote by $X_{1}, \ldots, X_{n}$ an i.i.d. real-valued sample drawn from an unknown probability density function (pdf) $P$ on $\mathbb{R}^{d}$. This sample may be transformed by some bijective function $f$, yielding an new sample $Y_{i}$ such that $Y_{j}=f\left(X_{j}\right)$ that may be considered to be drawn from an other probability density function (pdf) $Q$ on $\mathbb{R}^{d}$.

\subsection{Sample and Continuous Probability Density Functions}

Estimating $P$ is a well known problem. If this function has a parametric form (e.g. mixture of Gaussians), classical estimation techniques, such that the maximum likelihood of the function's parameters, may work. But, in most cases, one may consider it as non-parametric. Here, $P$ can be estimated by $P_{s}$ by the AkaikeParzen-Rosenblatt windowing technique Aka54 Par62|Ros56.

$$
P_{s}(x)=\frac{1}{n} \sum_{j=1}^{n} K_{s}\left(x-X_{j}\right)
$$

where $s>0$ is a smoothing factor, $K$ is a non negative absolutely integrable function (called the kernel).

The choice of both the kernel and the smoothing factor is still a research topic BD94], but will not be discussed here. Our particular choice (somewhat 
classical) for the kernel function is the Gaussian, and the smoothing factor is then $\sigma$.

As we are only interested in 1-D discrete probability functions, we will from now on restrict our study for $d=1$.

\subsection{Samples, Histograms, and Discrete Probability Density Functions}

An histogram $h$ can be built from the sample by grouping the $X_{j}$ into bins $b_{i}$, each of them being represented by a value $x_{i}$. In the 1-D case, the bins are 1-D intervals. When dealing with gray-scale images, usually encoded with integers at the acquisition stage, the $x_{i}$ are integer values, and the bin width is obviously 1 .

It can be shown that the optimal (under certain conditions) boundary between two consecutive bins $x_{i}$ and $x_{i+1}$ is given by $\left(x_{i}+x_{i+1}\right) / 2$ MGC01Llo57.

Although the sampling of the $x_{i}$ can be adapted to correspond to a better perception of the images [MGC01], we will here restrict ourself to a regular sampling (i.e. $\left|x_{i+1}-x_{i}\right|=$ cste). Moreover, the choice of the optimal bin's width could naturally be a research subject Wan97] that will not be investigated here.

Let us now consider that the histogram is defined by some points $x_{i}$, and that the frontier between two consecutive points $x_{i}$ and $x_{i+1}$ is located in the middle $\left(x_{i}+x_{i+1}\right) / 2$. Equivalently, we can said that each bin $x_{i}$, with $i=0 \ldots I$, is defined by the interval $\left[x_{i}^{\text {min }}, x_{i}^{\max }\right]$ where $x_{i}^{\text {min }}=\left(x_{i-1}+x_{i}\right) / 2$ and $x_{i}^{\max }=$ $\left(x_{i}+x_{i+1}\right) / 2$. To be consistent, we define $x_{-1}=-\infty$ and $x_{I+1}=+\infty$.

The histogram $h$ is thus defined by a finite number of integer values $h\left(x_{i}\right)$. From it, it is straightforward to build a discrete probability density function, $p\left(x_{i}\right)$ by normalizing by the sample size $n=\sum_{i} h\left(x_{i}\right): p\left(x_{i}\right)=1 / n h\left(x_{i}\right)$.

\subsection{Discrete and Continuous Probability Density Functions}

From above, it comes out that estimating a continuous probability density function from a discrete one can be achieved by the Akaike-Parzen-Rosenblatt windowing technique. It comes to consider that all the $X_{j}$ falling into a bin $b_{i}$ are located at $x_{i}$.

$$
P_{s}(x)=\sum_{i=0}^{I} p\left(x_{i}\right) K_{s}\left(x-x_{i}\right)
$$

Building a discrete pdf from a continuous one is also straightforward $p\left(x_{i}\right)=$ $\int_{x_{i}^{\text {min }}}^{x_{\text {max }}} P(x)$. Thus, new (smoothed) discrete pdfs (defined by values $y_{j}$ and bins $\left.\left[y_{j}^{\min } y_{j}^{\max }\right]\right)$ can be easily deduced from a given one, through Parzen windowing, i.e.

$$
\tilde{p}_{s}\left(y_{j}\right)=\int_{y_{j}^{\min }}^{y_{j}^{\max }} P_{s}(x) d x=\sum_{i=0}^{I} p\left(x_{i}\right) \int_{y_{j}^{\min }-x_{i}}^{y_{j}^{\max }-x_{i}} K_{s}(x) d x
$$

It comes out that $\tilde{p}_{s}\left(y_{j}\right)$ can be expressed as a linear combination of the $p\left(x_{i}\right)$. It should be pointed out that equation 2 can be used for intensity transformation, 
or bin's width changes, or also to regularize the discrete probability density function $p\left(x_{i}\right)$ by

$$
p_{s}\left(x_{j}\right)=\sum_{i=0}^{I} p\left(x_{i}\right) \int_{x_{j}^{\text {min }}-x_{i}}^{x_{j}^{\max }-x_{i}} K_{s}(x) d x
$$

Of course, previous expressions can be simplified if an analytical form of a primitive function if known. For example, if the Gaussian is chosen as kernel, it leads to $\tilde{p}_{s}\left(y_{j}\right)=\frac{1}{2} \sum_{i=0}^{I} p\left(x_{i}\right)\left(\operatorname{erf}\left(\frac{y_{j}^{\text {max }}-x_{i}}{\sqrt{2} \sigma}\right)-\operatorname{erf}\left(\frac{y_{j}^{\text {min }}-x_{i}}{\sqrt{2} \sigma}\right)\right)$.
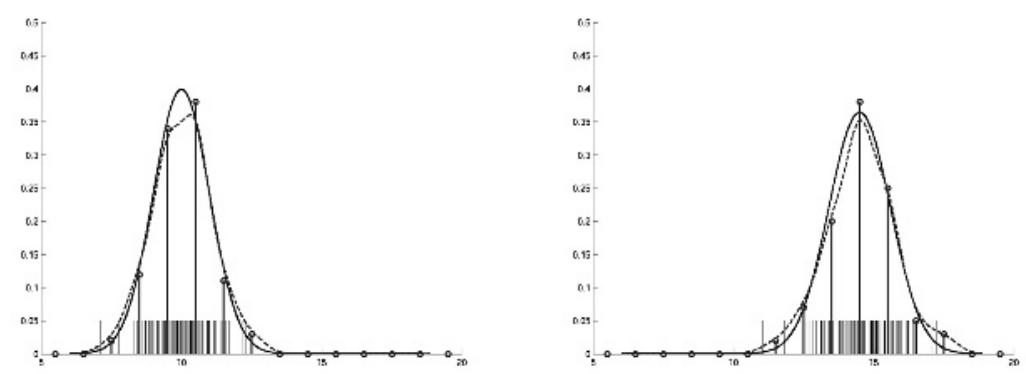

Fig. 1. A sample $(n=100)$ drawn from a Gaussian distribution (small vertical segments at the left) has been transformed by an affine function $y=1.2 x+2.5$ (at the right). Continuous curves: original Gaussian distributions; vertical segments topped by circles: discrete pdfs computed from the samples; dashed curves: continuous pdfs estimated from the discrete ones (equation $10=0.5$ ).

\section{Histogram Matching}

Consider two discrete histograms or equivalently two discrete pdfs $p\left(x_{i}\right)$ and $q\left(y_{j}\right)$. Histogram matching consists in computing the best function $\hat{f}, y=f(x)$, with respect to some similarity measure $S: \hat{f}=\arg \min _{f \in \mathcal{F}} S\left(p\left(x_{i}\right), q\left(y_{j}\right), f\right)$. As $p\left(y_{j}\right)$ and $q\left(x_{i}\right)$ are not directly comparable, we transform the above problem to

$$
\left.\hat{f}=\arg \min _{f \in \mathcal{F}} S\left(\tilde{p}_{s}\left(x_{i}\right), \widetilde{(q \circ f)}\right)_{s}\left(x_{i}\right)\right)
$$

where $\tilde{p}_{s}\left(x_{i}\right)$ comes from equation 3 and

$$
\widetilde{(q \circ f)_{s}}\left(x_{i}\right)=\sum_{j=0}^{J} q\left(y_{j}\right) \int_{f\left(x_{i}^{\min }\right)-y_{j}}^{f\left(x_{i}^{\max }\right)-y_{j}} K_{s}(u) d u
$$

is the discrete pdf built at each value $x_{i}$ by integrating from $f\left(x_{i}^{\text {min }}\right)$ to $f\left(x_{i}^{\text {max }}\right)$ the continuous pdf deriving from the $q\left(y_{j}\right)$. This way, we build a (smoothed) discrete $\operatorname{pdf} \tilde{q}_{s}\left(x_{i}\right)$ from $q\left(y_{j}\right)$ at the same values $x_{i}$ than the ones of $p\left(x_{i}\right)$, but 
also with consistent bin widths. This last characteristic is the main contribution of our approach.

Figure 1 presents the discrete pdfs, $q\left(y_{j}\right)$ and $p\left(x_{i}\right)$ of one sample drawn from a Gaussian distribution, with and without being transformed by an affine function. The same bin width is used to compute both discrete pdfs.

Applying above formulas to get both $\tilde{p}_{s}\left(x_{i}\right)$ and $\widetilde{(q \circ f)}\left(x_{i}\right)$, the similarity between the two original discrete pdfs, $p\left(x_{i}\right)$ and $q\left(y_{j}\right)$, can then be more easily estimated (see figure 21) by the similarity between $p\left(x_{i}\right)$ and $\widetilde{(q \circ f)}{ }_{s}\left(x_{i}\right)$, or preferably between $\tilde{p}_{s}\left(x_{i}\right)$ and $\widetilde{(q \circ f)_{s}}\left(x_{i}\right)$.

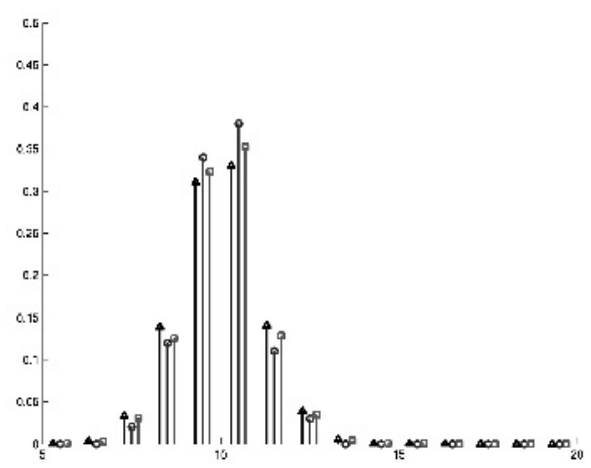

Fig. 2. Segments topped by circles: the original discrete pdf $p\left(x_{i}\right)$; segments topped by squares: the smoothed discrete pdfa $\widetilde{(q \circ f)})_{s}\left(x_{i}\right)$ estimated from the $q\left(y_{j}\right)$; segments topped by triangles: the smoothed original discrete pdf $\tilde{p}_{s}\left(x_{i}\right)$.

As the above criteria is not symmetrical, we prefer to change it into

$$
\left.\hat{f}=\arg \min _{f \in \mathcal{F}}\left(S\left(\tilde{p}_{s}\left(x_{i}\right), \widetilde{(q \circ f}\right)_{s}\left(x_{i}\right)\right)+S\left(\left(\widetilde{p \circ f^{-1}}\right)_{s}\left(y_{j}\right), \tilde{q}_{s}\left(y_{j}\right)\right)\right)
$$

when $f$ is invertible.

Our practical choices are to use parametric functions $f$ (polynomials), and minimization is done with a classical Powell-Brent procedure. We implemented several similarity measures, from the sum of squared differences to the maximum of the likelihood.

\section{Results}

\subsection{Monkey's Brain Autoradiographies}

The MAPAWAMO european project aims at mapping visual cortical regions in awake, behaving monkey using functional MR images (fMRI). One of the innovative aspects of this project is to compare within the same monkey activation maps measured with fMRI and those obtained by metabolic labelling (double label 2-deoxyglucose technique VTO00), thus allowing to compare two mapping techniques with each other, the 2DG serving as "ground truth", and advance the understanding of the nature of the fMRI signal. 

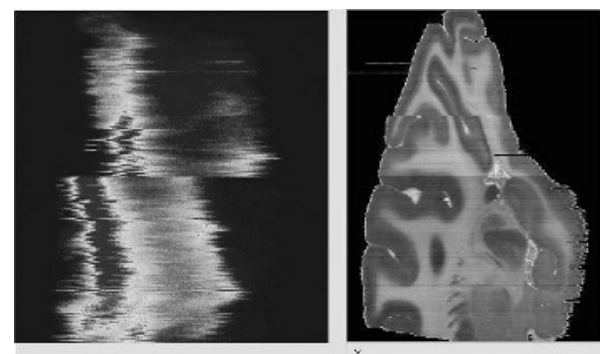

120

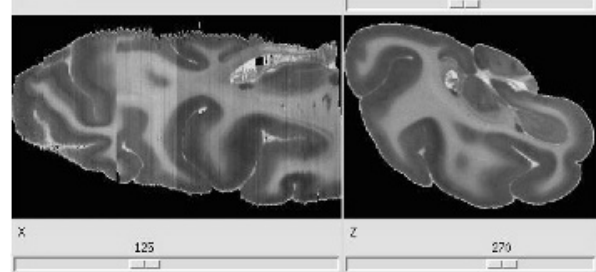

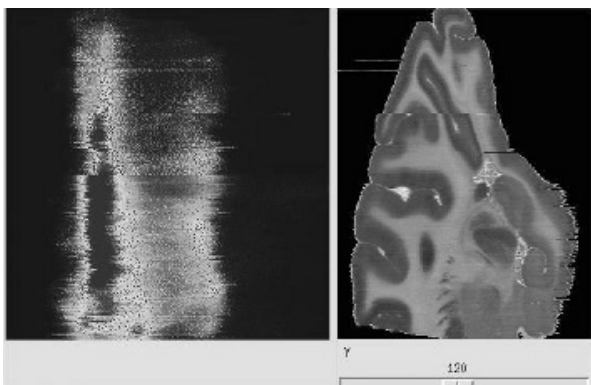

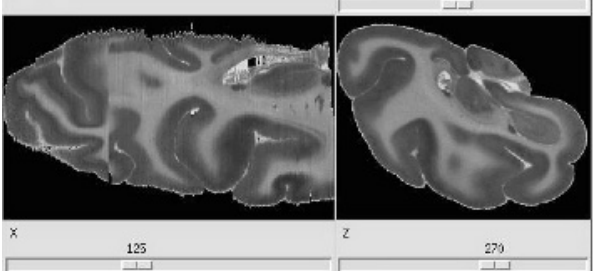

Fig. 3. Monkey's brain autoradiographies. Three orthogonal slices of the realigned stack of 2D autoradiographies before (left) and after (right) intensity normalisation. Upper left images: histograms of contiguous sections (viewed as an elevation surface from above, each line of these figures corresponding to the histogram of a given section; blue and red correspond respectively to lowest and highest probabilities; the main left strip corresponds to grey matter, the right strip to white matter) before (left) and after (right) intensity normalisation.

Acquisition of the 2DG data was carried out by autoradiography, after monkey's sacrifice and histological procedure, leading to series of 417 contiguous $40 \mu \mathrm{m}$ thin sections. The first task consisted in the realignment of these disorganized serial sections into a geometrically consistent volume. This was carried out using the block matching algorithm $\mathrm{ORS}^{+} 01, \mathrm{MB} 03$, an intensity-based iterative two-steps method consisting in selective local-based correspondance computation, followed by robust transformation estimation. Due to the acquisition and digitalisation processes, variations in intensity from slice to slice were observed in the reconstructed volume (Fig. 3) Therefore intensity normalisation was needed before further 3-D analysis of this volume, as activations result in intensity hyposignals in cortical regions. Normalisation was performed with the methodology presented in this paper (see Section [3), using an affine function, the symmetrical sum of squared differences as similarity measure, with Gaussian kernel sigma set to 5.0.

\subsection{Human Brain Optical Sections}

In functional neurosurgery, there is a need for accurate localisation of the functional targets. The SIERA project aims at constructing a three dimensional, anatomical and functional, as well as registrable, cartography of the basal ganglia, based on histology $\mathrm{BOM}^{+} 02$. For doing this, a post mortem MR study was conducted on a cadaver's head, 36 hours after death, and the brain was 
then extracted and processed for histology. Fusion of MR and histological data will allow to report this cartography on the patient's anatomy, by its registration with the patient's MR image.
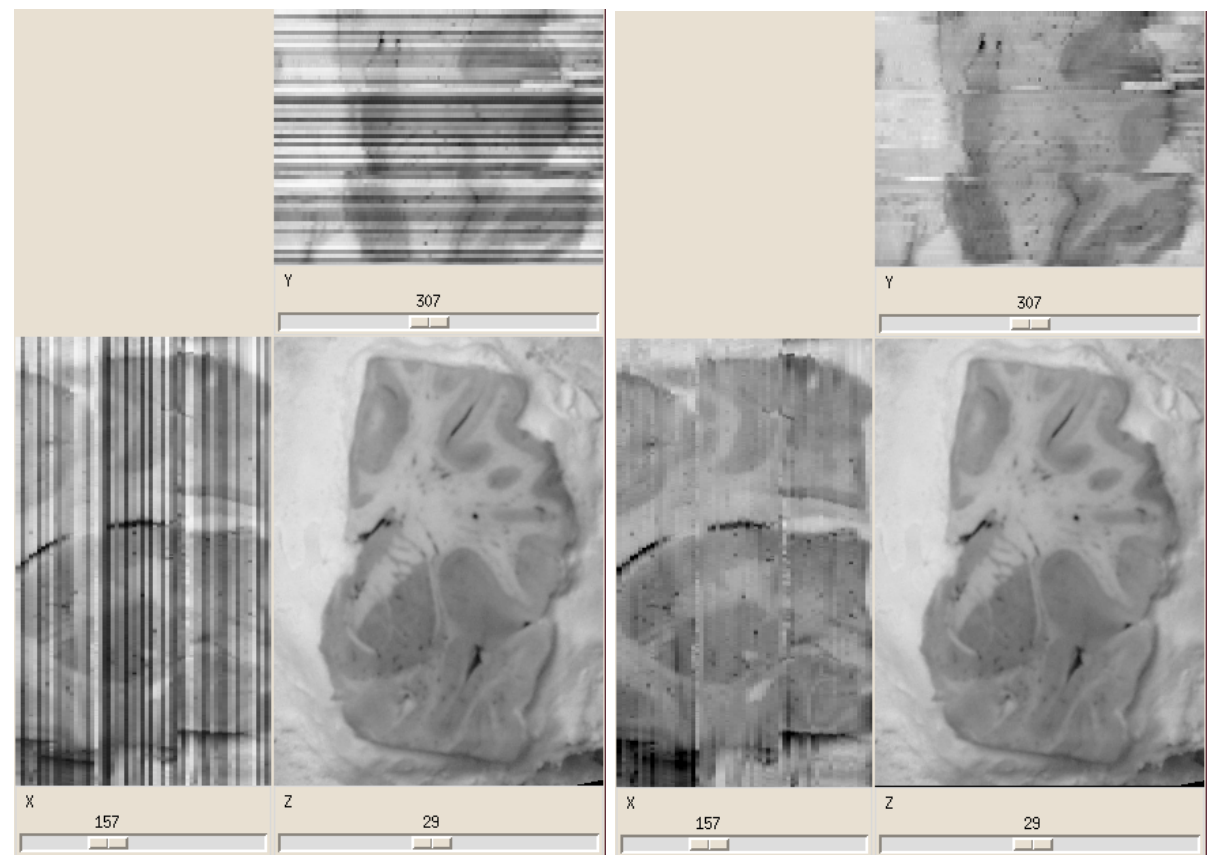

Fig. 4. Three orthogonal slices of the realigned stack of $2 \mathrm{D}$ optical sections of the human brain before (left) and after (right) intensity correction.

One hemisphere of the brain was stored frozen at $-40^{\circ} \mathrm{C}$ and cut into $70 \mu \mathrm{m}$ thin sections which were collected serially. Sectioning was done on a Tetrander Jung freezing microtome and photographs of the unstained surface of the frozen brain were taken for one out of ten sections. As for monkey's autoradiographies, realignment of these optical sections was done with the block matching algorithm [ORS ${ }^{+}$01]. Large variations in intensity were observed in the reconstructed volume, due to acquisition and scanning of the optical data. Therefore intensity normalisation was needed before further intensity-based registration with the post mortem MR images. Normalisation was similarly performed using an affine function, the symmetrical sum of squared differences as similarity measure, with Gaussian kernel sigma set to 5.0.

\section{Conclusion}

In this work, we have developed a formal framework to compare discrete probability density functions. Applying an intensity function to an discrete pdf $p\left(x_{i}\right)$ was then not done by applying the function to the discrete intensities $x_{i}$ as usual, 
but to the bins of the histogram leading to a more consistent histogram transformation. We have based our approach on windowing techniques. This way, we used implicitly an estimated continuous pdf, which allowed us to get rid of intensity discretisation problems.

We have presented results on real data, monkey's brain autoradiographs and human brain optical sections. With the proposed method, we obtained homogeneous reconstructions: it allows first a visual qualitative inspection of the reconstructed volume, and second to use this volume for a further registration with 3-D corresponding images (e.g. MRI).

Methodological developments will include multi-scale approach, by changing the bin's width, and more detailed study of similarity measures (surprisingly, we found out that the sum of squared differences seemed to perform better than other, and more evolved, implemented measures).

Acknowledgments. Authors are very grateful to Alexis Roche and David Rey for numerous and stimulating exchanges. This work was partially funded by European project MAPAWAMO (ref. QLG3-CT-2000-30161; coordinator: Pr. Guy Orban, Lab. of Neuro- and Psychophysiology, Dept. of Neurosciences and Psychiatry, K.U. Leuven, Belgium). We thank W. Vanduffel, K. Nelissen and G. Orban for providing us with the monkey's autoradiographs, and Dr. J. Yelnik (INSERM U.289, Paris) for the human brain optical images.

\section{References}

[Aka54] H. Akaike. An approximation to the density function. Annals of the Institute of Statistical Mathematics, 6:127-132, 1954.

[BD94] A. Berlinet and L. Devroye. A comparison of kernel density estimates. Publications de l'Institut de Statistique de l'Université de Paris, 38(3):359, 1994. http://jeff.cs.mcgill.ca/ luc/np.html.

$\left[\mathrm{BOM}^{+} 02\right]$ E. Bardinet, S. Ourselin, G. Malandain, D. Tandé, K. Parain, N. Ayache, and J. Yelnik. Three dimensional functional cartography of the human basal ganglia by registration of optical and histological serial sections. In IEEE International Symposium on Biomedical Imaging, 2002.

[Cas96] Kenneth R. Castleman. Digital Image Processing, chapter Point Operations, pages 83-97. Prentice Hall International Editions, 1996.

[Hea96] C.F. Hildebolt and R.K. Walkup et al. Histogram-matching and histogram-flattening contrast correction methods: a comparison. Dentomaxillofac. Radiol., 25(1):42-47, 1996.

[Llo57] S.P. Lloyd. Least squares quantization in PCM's. Laboratories paper, Bell Telephone, Murray Hill, NJ, 1957.

[MB03] G. Malandain and E. Bardinet. Fusion of autoradiographies with an mr volume using 2-d and 3-d linear transformations. In Proc. of IPMI'03, pages 487-498, 2003.

[MGC01] Cicero Mota, Jonas Gomes, and Maria I.A. Cavalcante. Optimal Image Quantization, Perception and the Median Cut Algorithm. An. Acad. Bras. Cienc., 73(3):303-317, 2001.

[MV98] J.B.A. Maintz and M.A. Viergever. A survey of medical image registration. Medical Image Analysis, 2(1):1-36, march 1998. 
$\left[\mathrm{ORS}^{+}\right.$01] S. Ourselin, A. Roche, G. Subsol, X. Pennec, and N. Ayache. Reconstructing a 3D Structure from Serial Histological Sections. Image and Vision Computing, 19(1-2):25-31, January 2001.

[Par62] E. Parzen. On the estimation of a probability density function and the mode. Annals of Mathematical Statistics, 33:1065-1076, 1962.

[Ros56] M. Rosenblatt. Remark on some nonparametric estimates of a density function. Annals of Mathematical Statistics, 27:832-837, 1956.

[RSMA01] D. Rey, J. Stoeckel, G. Malandain, and N. Ayache. Using SPM to detect evolving MS lesions. In Proceedings of MICCAI'01, volume 2208 of LNCS, pages 1232-1234, Utrecht, The Netherlands, October 2001.

[VTO00] W. Vanduffel, R.B.H. Tootell, and G.A. Orban. Attention-dependent suppression of metabolic activity in the early stages of the macaque visual system. Cerebral Cortex, 10:109-126, 2000.

[Wan97] M.P. Wand. Data-Based Choice of Histogram Bin Width. The American Statistician, 51(1):59-64, February 1997.

[WLB ${ }^{+}$98] Liqun Wang, H-Ming Lai, Gareth J. Berker, David H. Miller, and Paul S. Tofts. Correction for Variations in MRI Scanner Sensitivity In Brain Studies with Histogram Matching. Magn. Reson. Med, 39:322-327, 1998. 